\title{
Consolidating and Exploring Information via Textual Inference
}

\author{
Ido Dagan \\ Computer Science Department \\ Bar-Ilan University \\ dagan@cs.biu.ac.il
}

Effectively consuming information from large amounts of texts, which are often largely redundant in their content, is an old but increasingly pressing challenge. It is well illustrated by the perpetual attempts to move away from the flat result lists of search engines towards more structured fact-based presentations. Some recent attempts at this challenge are based on presenting structured information that was formulated according to pre-defined knowledge schemes, such as Freebase and Google's knowledge graph. We propose an alternative, as well as complementary, approach that attempts to consolidate and structure all textual statements in a document collection based on the inference relations between them. Generic textual inference techniques, formulated under the Textual Entailment paradigm, are used to consolidate redundant information into unique "core" statements, and then present them in an intuitive general-to-specific hierarchy. The talk will review some of the underlying concepts and algorithms behind our approach and present an initial demo.

The original version of this chapter was revised: The copyright line was incorrect. This has been corrected. The Erratum to this chapter is available at DOI: 10.1007/978-3-319-02432-5_33

O. Kurland, M. Lewenstein, and E. Porat (Eds.): SPIRE 2013, LNCS 8214, p. 1, 2013.

(C) Springer-Verlag Berlin Heidelberg 2013 